\title{
MILLIMETER-WAVE MONITORING OF NUCLEAR WASTE GLASS MELTS - AN OVERVIEW
}

\author{
P. P. Woskov, J. S. Machuzak, and P. Thomas \\ Plasma Science and Fusion Center \\ Massachusetts Institute of Technology \\ Cambridge, MA 02139 \\ S. K. Sundaram \\ Pacific Northwest National Laboratory \\ Richland, WA 99352
}

William E. Daniel, Jr.

Westinghouse Savannah River Company

Aiken, SC 29808

\begin{abstract}
Molten glass characteristics of temperature, resistivity, and viscosity can be monitored reliably in the high temperature and chemically corrosive environment of nuclear waste glass melters using millimeter-wave sensor technology. Millimeter-waves are ideally suited for such measurements because they are long enough to penetrate optically unclear atmospheres, but short enough for spatially resolved measurements. Also efficient waveguide and optic components can be fabricated from refractory materials such as ceramics. Extensive testing has been carried out at a frequency of $137 \mathrm{GHz}$ to temperatures up to $1500{ }^{\circ} \mathrm{C}$. Performance of refractory waveguides at high temperature has been shown to be satisfactory. A novel new method for viscosity monitoring has also been tested with simulated nuclear waste glasses. It has been shown that a viscosity range of over 30 to 3000 Poise can be monitored with one instrument. Results of these laboratory tests and the potential of millimeter-wave sensors for on-line glass process monitoring are presented.

\section{INTRODUCTION}

The vitrification and long term storage of high level and low activity nuclear waste represents one of the major challenges of the U. S. DOE Environmental
\end{abstract}


Management's effort to clean up nuclear waste sites. Optimization of the vitrification process through improved process control could significantly reduce costs associated with this effort. Improved process control would result in increased glass manufacturing efficiencies, reduced storage volumes through increased waste loading, and reduced storage risk by insuring the long stability quality of the poured glass product.

Present nuclear waste melters such as the Defense Waste Processing Facility (DWPF) and planned melters for the Hanford site lack sophisticated diagnostics due to the hot, corrosive, and radioactive environments of these melters. New robust diagnostics are needed that can operate on-line in these environments. Parameters important for monitoring include temperature profiles of the glass melt, cold cap, plenum off gases, molten glass conductivity, density, and viscosity. With real time monitoring of these parameters it should be possible to implement feedback control of the vitrification process to achieve significant cost savings.

Much of the desired on-line monitoring capabilities can be realized through the use of millimeter-wave (MMW) technologies. Electromagnetic radiation in the $10-0.3 \mathrm{~mm}(30-1000 \mathrm{GHz})$ range of the spectrum is ideally suited for remote measurements in harsh, optically unclean and unstable processing environments. Millimeter waves are long enough to penetrate optical/infrared obscured viewing paths through dust, smoke, and debris, but short enough to provide spatially resolved point measurements for profile information. Another important advantage is the ability to fabricate efficient MMW melter viewing components from refractory materials. Application of MMW pyrometry to a dc arc furnace melter has been previously demonstrated [1].

\section{MILLIMETER-WAVE SENSOR APPROACH}

The millimeter-wave sensor approach is illustrated in Figure 1. A sensitive MMW receiver is used to view the molten gas via a quasi-optical transmission line. The receiver both receives signals from the glass and transmits a MMW probe signal to monitor reflections. The transmission line makes use of waveguides and can have one or more optical elements such as a mirror. The transmission line is highly efficient making possible remote placement of the MMW receiver outside of any biological shield around the melter facility. Profile measurements are made possible by appropriate design of the waveguide components inside the melter for rotation and/or translation [1]. 


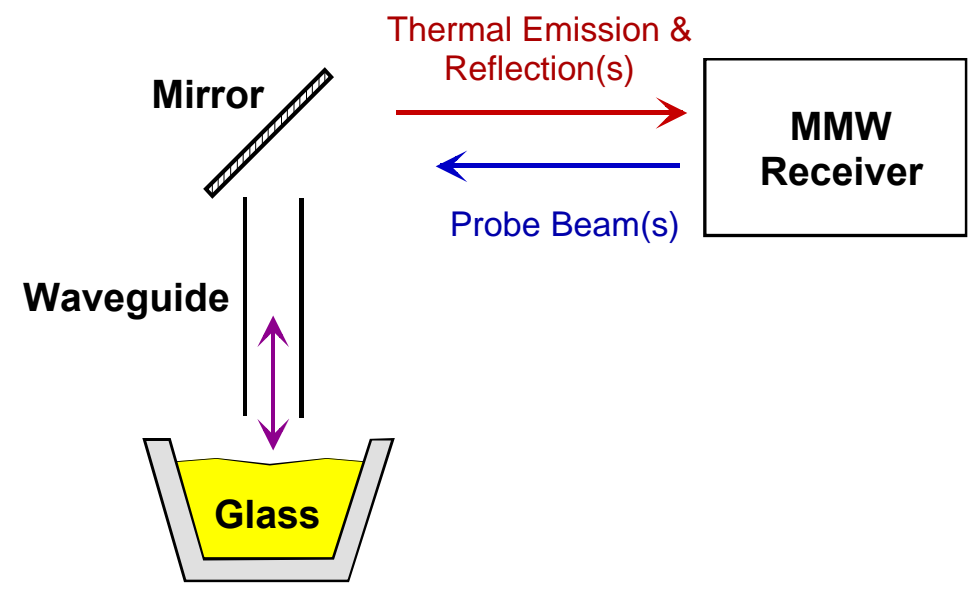

Figure 1. Millimeter-wave sensor configuration

The physical molten glass parameters that would be measured and the corresponding millimeter-wave phenomena that are exploited to achieve these measurements are listed in Table I. Temperature is determined by detecting the thermal emission from the viewed glass. The reflectivity of the glass, which can be used to determine the glass electrical resistively or conductivity [2] is obtained from measuring the millimeter-wave reflection amplitude. Glass density is determined by measuring reflection phase change in response to a pressure displacement. The reflection phase change gives the glass fluid displacement, which depends on density for a given pressure. Viscosity is then determined from the rate of phase change in response to a given pressure transient.

Table I. MMW Measurements

\begin{tabular}{ll}
\hline Parameter & Measured Effect \\
\hline Temperature (T) & Thermal emission \\
Reflectivity $(r)$ & Reflection amplitude \\
Density $(\rho)$ & Reflection phase \\
Viscosity $(\eta)$ & Reflection phase rate \\
\hline
\end{tabular}

\section{BASIS FOR TEMPERATURE MEASUREMENT}

The temperature of the viewed molten glass is given by the thermal emission signal. Unlike commercial pyrometers, which operate in the infrared portion of the electromagnetic spectrum, millimeter-wave thermal emission is linear with temperature. The thermal emission is given by the blackbody emission formula for a single polarization in this wavelength limit as [3]: 


$$
P=k_{B} T_{e f f} d v
$$

where $P$ is in Watts, $k_{B}$ is Boltzmann's constant, $T_{\text {eff }}$ is the absolute temperature in Kelvin that fills the receiver antenna, and $d v$ is the receiver bandwidth in Hertz. The receiver signal is linearly proportional to $T_{e f f}$, which facilitates calibration and gives a MMW pyrometer a much larger dynamic range than possible in the infrared.

The effective temperature that fills the receiver antenna depends on the efficiency of the transmission line and the emissivity of the sample of molten glass that is viewed. All sources and losses of thermal radiation need to be considered when determining $T_{\text {eff. }}$. They are identified in Figure 2. $T_{\text {eff }}$ is given by the formula:

$$
T_{e f f}=\varepsilon_{w g} T_{w g}+\tau_{w g} \varepsilon_{s} T_{s}+r_{s} \tau_{w g} \varepsilon_{w g} T_{w g}
$$

where the first term on the right is the thermal emission from the waveguide, the second term is the thermal emission from the glass, and the finial term is the reflected thermal emission from the waveguide, where $\varepsilon_{w g}$ is the waveguide emissivity, $\varepsilon_{s}$ is the sample glass emissivity, $\tau_{w g}$ is the waveguide transmission factor, $r_{s}$ is the sample glass reflectivity, and $T_{w g}$ and $T_{s}$ are the temperatures of the waveguide and sample glass, respectively.

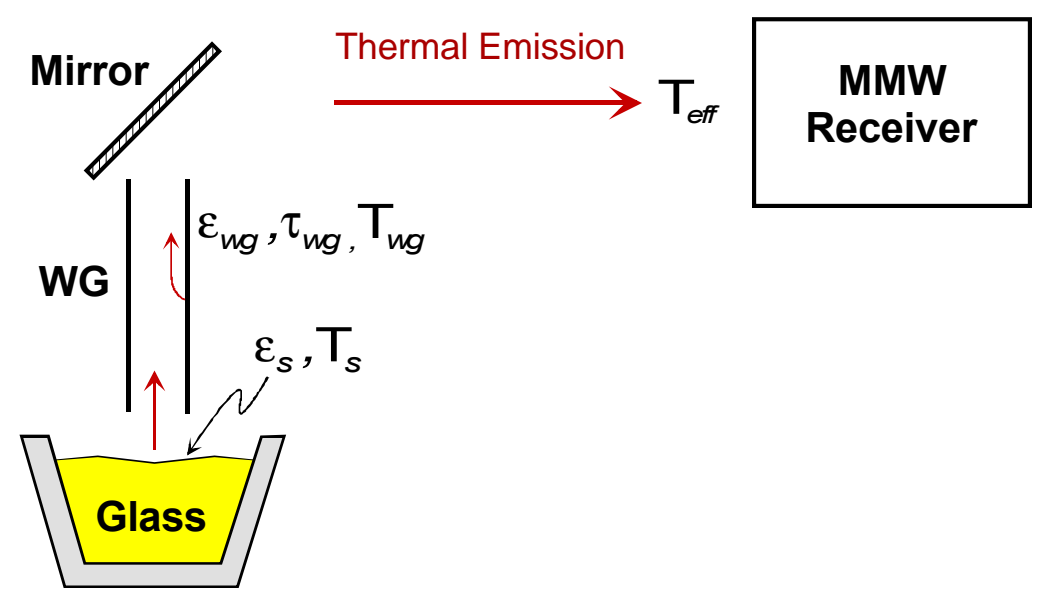

Figure 2. MMW receiver temperature measurement

It can be shown that the waveguide emissivity and transmission factor, and the sample glass emissivity and reflectivity are related by the expressions: 


$$
\begin{gathered}
\varepsilon_{w g}=\left(1-\tau_{w g}\right) \\
\varepsilon_{s}=\left(1-r_{s}\right)
\end{gathered}
$$

When the transmission line has no losses $\left(\tau_{w g} \approx 1\right)$ and the sample glass is near a prefect blackbody $\left(\varepsilon_{s} \approx 1\right)$, the receiver signal is representative of the glass temperature, $T_{\text {eff }} \approx T_{s}$. In other cases the other parameters in Equation 2 must be considered in determining temperature.

\section{TECHNIQUE FOR REFLECTION MEASUREMENT}

In most cases it is important to know the glass reflectivity (emissivity) not only to determine temperature accurately, but also to provide information of the electrical resistivity of the glass. A MMW reflection measurement is accomplished by redirecting a portion of the thermal signal back at the viewed sample. Using the broadband thermal signal as a probe of reflectivity avoids the standing wave effects of a coherent probe beam [1].

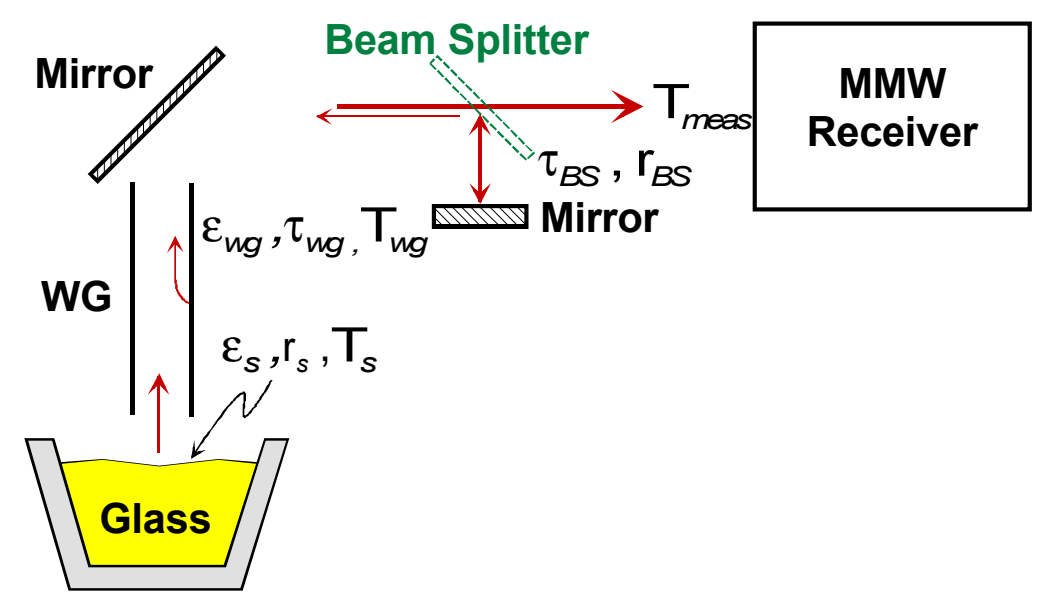

Figure 3. Thermal return reflection setup for reflectivity measurements

A thermal return reflection measurement is implemented by using a beam splitter in front of the receiver to divert some of the thermal signal from the melter to a side mirror as shown in Figure 3. When the side mirror is blocked to prevent reflection, the receiver signal is just the temperature signal, $T_{\text {eff, }}$ reduced by the beam splitter transmission factor, $\tau_{b s}$. When the side mirror is unblocked to return a signal to the sample the receiver signal increases by an amount dependent on the sample reflectivity. The view of the sample is aligned so that a signal incident from the waveguide is reflected back up the waveguide. The measured temperatures with the side mirror blocked and unblocked can be expressed as: 


$$
\begin{gathered}
T_{\text {meas }}=\tau_{b s} T_{e f f} \\
T_{\text {meas }}^{\prime}=\tau_{b s} T_{e f f}+r_{b s} T_{e f f}\left(1-r_{s} \tau_{b s}^{2} \tau_{w g}^{2}\right)^{-1}
\end{gathered}
$$

By taking these two temperature measurements, one with the side mirror blocked and another with this mirror unblocked, it is possible to solve for the viewed sample reflectivity, $r_{s}$. This is expressed as:

$$
r_{s}=\frac{1}{\tau_{B S}^{2} \tau_{w g}^{2}}\left(1-\frac{T_{\text {meas }}}{T_{\text {meas }}^{\prime}}\right)
$$

Equation 7 with Equations 4 and 2 along with knowledge of the waveguide and beam splitter transmission factors makes possible the determination of both temperature and emissivity of the viewed molten glass.

\section{BASIS FOR POSITION AND FLOW MEASUREMENTS}

A MMW receiver used in practice for temperature measurements is a heterodyne receiver, that is, it uses a MMW local oscillator to frequency down shift the thermal radiation for detection with a conventional diode detector. Molten glass position and flow measurements are accomplished by using the leaked local oscillator signal from the antenna toward the viewed sample. This is a coherent signal and consequently the reflected phase can be determined. Reflected phase is an indicator of the distance to the reflecting surface from the receiver. The phase will cycle through 180-degree phase shifts for every $1 / 4$ wavelength shift in distance between the receiver and reflecting surface. The phase is expressed as:

$$
\delta=4 \pi \frac{\Delta y}{\lambda}
$$

where $\Delta y$ if the molten glass displacement and $\lambda$ is the local oscillator wavelength. A quadrature receiver or amplitude cycling from a single mixer receiver could be used to detect this phase.

For the position and flow measurements the waveguide is immersed into the molten glass and sealed with a window at the other end (as shown in Figure 4). This allows the waveguide to be pressurized to control the position and flow of the glass inside the waveguide. The absolute displacement of the glass divided into the displacement pressure in terms of water displacement gives the glass density directly. Viscosity is determined by inducing a sudden pressure transient in the waveguide and monitoring the velocity of the molten glass flow as manifested by the rate of change of the reflection phase. 


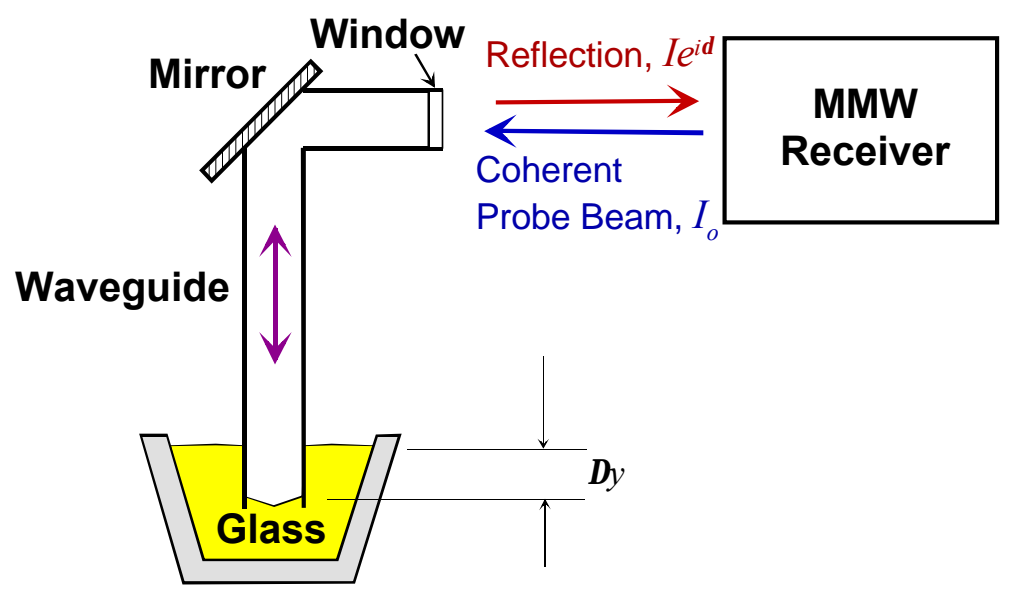

Figure 4. MMW position and flow measurement setup

\section{REFRACTORY WAVEGUIDES}

Efficient hollow millimeter-wave waveguides can be fabricated from refractory materials that can be used in high temperature environments. The transverse electric and magnetic field $\mathrm{HE}_{11}$ mode is the most efficient guided mode for electromagnetic propagation in hollow waveguides with internal diameter larger than a wavelength [4]. This mode is also an optimum for launching a diffraction-limited free space Gaussian beam, ideal for most diagnostic applications. The $\mathrm{HE}_{11}$ mode is a natural mode inside smooth walled dielectric tubes and can be achieved inside electrically conducting metallic tubes by circumferentially corrugating the inside wall with $1 / 4 \lambda$ deep grooves at more than 2.5 grooves per $\lambda$ of waveguide length. For typical metallic resistivities and dielectric indexes of refraction, smooth walled dielectric waveguides need to be much larger diameter to achieve the same transmission efficiencies as corrugated waveguides.

A number of different refractory waveguides were fabricated and tested at 137 GHz. Table II lists the types of waveguides tested, diameter, wall thickness, and measured transmission efficiency. Inconel 690 was corrugated with a 32/inch $(1.26 / \mathrm{mm})$ screw tap with V-shaped grooves. The measured losses of about $5.6 \%$ were higher than predicted by theory $(<1 \%)$ probably because the grooves did not machine well. After more than 30 hours of repeated use up to $1100{ }^{\circ} \mathrm{C}$ and then several hours up to $1180^{\circ} \mathrm{C}$ the Inconel developed a black scaly oxide layer that increased transmission loses to $17 \%$. Most of the oxidation occurred during the short period of use at the higher temperature. 


\begin{tabular}{ccccc}
\hline Material & $\begin{array}{c}\text { Inside } \\
\text { Surface }\end{array}$ & Inside Diameter & Wall Thickness & $\begin{array}{c}\text { Measured } \\
\text { Transmission }\end{array}$ \\
\hline Inconel 690 & Corrugated & $28.6 \mathrm{~mm}$ & N/A & $94.6 \%$ \\
$\begin{array}{c}\text { Inconel } 690 \\
\text { after use to } \\
1180^{\circ} \mathrm{C}\end{array}$ & “ & “ & “ & $83.0 \%$ \\
$\begin{array}{c}\text { Vesuvius } \\
\text { Mullite }\end{array}$ & Smooth & $41.3 \mathrm{~mm}$ & $3.3 \mathrm{~mm}$ & $97.6 \%$ \\
$\begin{array}{c}\text { Coors } \\
\text { ( }\end{array}$ & & & \\
Silicon Carbide & Smooth & $41.3 \mathrm{~mm}$ & $6.4 \mathrm{~mm}$ & $87.5 \%$ \\
\hline
\end{tabular}

For higher temperature applications in oxidizing environments a ceramic, mullite $\left(3 \mathrm{Al}_{2} \mathrm{O}_{3} \cdot 2 \mathrm{SiO}_{2}\right)$, and silicon carbide were evaluated. Mullite tubes were acquired from two vendors, Vesuvius McDaniel and Coors. The Vesuvius ${ }^{\circledR}$ mullite had additional impurities in it to aid in the manufacture of drawn tubing which made the material more opaque at $137 \mathrm{GHz}$, a desirable quality for the walls of a MMW waveguide. The Coors ${ }^{\circledR}$ mullite was a pure material with the waveguide manufactured by a powdered process. The refractive index for both types was determined to be about 2.5 at $137 \mathrm{GHz}$ by interference measurements through flat plate samples.

The theoretically predicted transmission efficiency was $93 \%$ for these mullite waveguides. The measured transmission was higher for the Vesuvius ${ }^{\circledR}$ mullite and lower for the Coors ${ }^{\circledR}$ mullite suggesting that the walls were not thick enough to prevent interference form the outer waveguide surface in the Coors ${ }^{\circledR}$ sample. A silicon carbide waveguide from Carborundum of the same inner diameter as the mullite was also acquired. It was not a high resistivity dielectric and did not perform as well as the mullite.

\section{LABORATORY TEST SETUP}

Experimental millimeter-wave measurements of temperature, reflectivity, and glass flow were carried out on a crucible of molten glass inside an electrically heated furnace ${ }^{1}$. For these tests circular mullite waveguides were used, the 41.6 mm diameter Vesuvius ${ }^{\circledR}$ waveguide tested above was used for temperature and

${ }^{1}$ Deltech Model DT-31-RS-12 
reflectivity measurements and a smaller $28.6 \mathrm{~mm}$ diameter Vesuvius guide was used for flow measurements.

The mullite waveguide was mated by a $90^{\circ}$ steel miter bend to a circular corrugated brass waveguide of the same internal diameter. The refractory waveguide was inserted vertically into an electric furnace, as shown in Figure 5, where approximately two thirds of it was exposed to high temperatures. The brass part of the waveguide assembly, orientated horizontally above the furnace, facilitated the horizontal setup of the millimeter-wave receiver and antenna optics above and to the side of the furnace on an elevator platform. The elevator platform allowed raising and lowering the waveguide assembly with the electronics for waveguide immersion measurements into the glass.

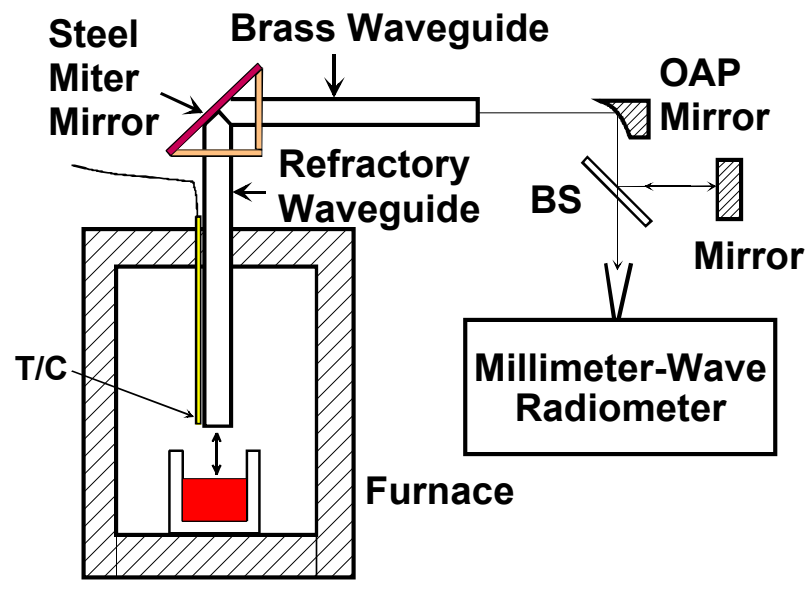

Figure 5. Experimental setup in the laboratory to test MMW senor methods

The receiver local oscillator frequency was $137 \mathrm{GHz}$ with an intermediate frequency range of $0.4-1.5 \mathrm{GHz}$. The scalar horn antenna field-of-view was coupled to the waveguide assembly $\mathrm{HE}_{11}$ mode by an off-axis parabolic (OAP) mirror. A quartz beam splitter (BS) and a flat side mirror in the antenna field-ofview allowed the use of the furnace thermal emission as a probe of the emissivity of the viewed surface inside the furnace. An S-type thermocouple inside the furnace provided an independent measure of temperature.

\section{EXPERIMENTAL TEMPERATURE AND EMISSIVITY MEASUREMENTS}

The capability of millimeter-wave pyrometry to measure temperature and emissivity was tested by viewing various surfaces at high temperature inside the furnace. Figure 6 shows the results for viewing a $1 / 4$ inch $(6.35 \mathrm{~mm})$ thick plate of Coors $^{\circledR}$ mullite resting on an alumina brick in the bottom of the furnace. The 
waveguide was lowered to within about $6 \mathrm{~mm}$ of the mullite surface to minimize diffraction of the return reflection.

The data is plotted as a function time in terms of the data acquisition count number and covers a period corresponding to several hours as the furnace was heated up, held at $1150{ }^{\circ} \mathrm{C}$, and then cooled down. The top trace shows the thermocouple temperature and the middle trace shows the MMW pyrometer temperature, $\mathrm{T}_{\text {meas. }}$ The pyrometer registers a lower temperature than the thermocouple because the waveguide has transmission losses and because the viewed mullite plate is not a perfect blackbody.

During the furnace temperature flat top at $1150{ }^{\circ} \mathrm{C}$, the pyrometer side mirror was blocked and unblocked several times to provide several measurements of $T_{\text {meas }}^{\prime}$. This part of the pyrometer temperature plot is shown expanded in the lower graph of Figure 6. The sharp dip in the pyrometer signal just after 10000 counts on the $\mathrm{x}$-axis was due to blocking the antenna for calibration.

Using Equation 7 and the measured waveguide transmission given in Table 2, the reflectivity of the viewed mullite plate at $1150{ }^{\circ} \mathrm{C}$ was calculated to be $r_{s}=$ 0.10. This corresponds to an emissivity of $\varepsilon_{s}=0.90$. An alternative determination of emissivity can be made by using the measured thermocouple temperature for $T_{s}$ in Equation 2 and solving for $\varepsilon_{s}$. The waveguide temperature can be approximated as a mean between the furnace and room temperatures. The emissivity computed in this way is plotted as the lowest trace in the upper graph of Figure 6.

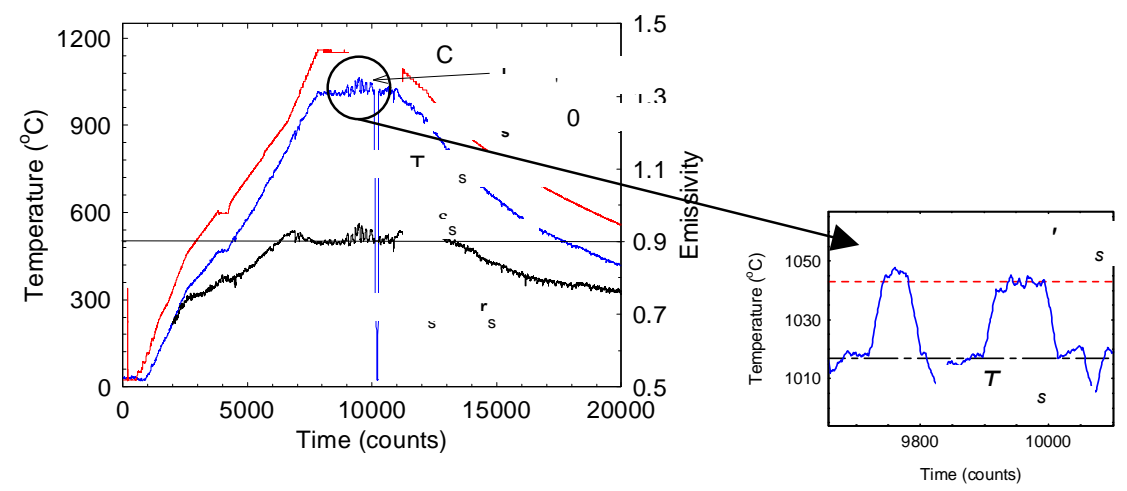

Figure 6. Measurements of temperature and emissivity of a mullite plate inside the furnace

At the temperature flat top the emissivity determined by the thermocouple and Equation 2 is in agreement with that calculated by Equation 7, as indicated by the straight line at $\varepsilon_{s}=0.90$. During the furnace heat up and cool down the 
emissivity determined by Equation 2 is not reliable because of the different time responses of the thermocouple and MMW receiver. Also, the emissivity that is measured here cannot be used to determine the dielectric properties of mullite because the mullite plate is too thin in this case to be totally opaque to $137 \mathrm{GHz}$ radiation.

The results of another test viewing an Inconel 690 plate inside the furnace are shown in Figure 7. As before the upper trace shows the thermocouple temperature and the lower trace is the measured millimeter-wave temperature. The millimeter-wave signal is very low because the Inconel plate is almost a perfect reflector. Applying the method of Equation 7 to the determination of emissivity results in a value of $\varepsilon_{s}=0.04$ during the $1150{ }^{\circ} \mathrm{C}$ temperature flat top. This value is actually too high because Inconel does not change its resistivity with temperature enough to cause the emissivity to go measurably above zero at 137 GHz. The most likely reason that the calculated emissivity deviates from zero is because the waveguide transmission losses have increased at high temperature. An increase of about $2 \%$ in $\tau_{w g}$ would account for the observed millimeter-wave thermal emission.

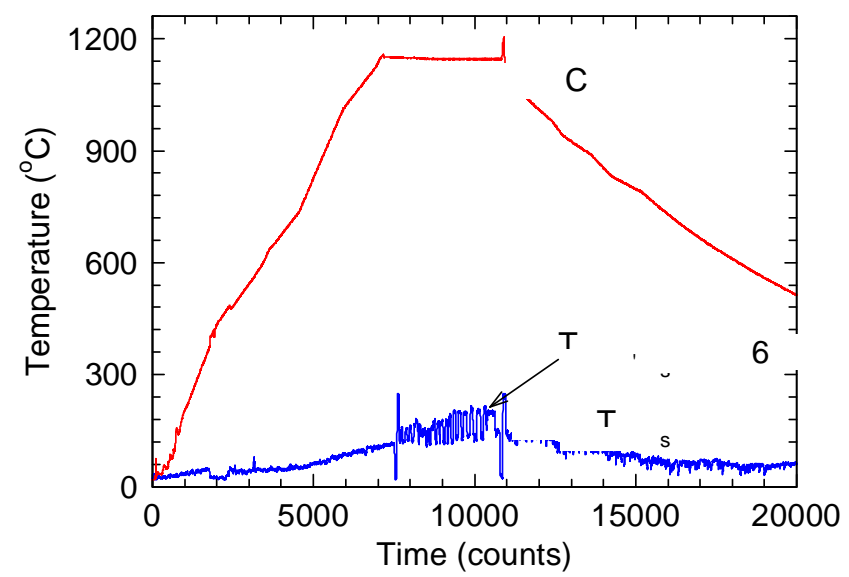

Figure 7. Temperature and emissivity measurements of an Inconel 690 plate

\section{EXPERIMENTAL FLOW MEASUREMENTS}

Molten glass flow measurements inside a sealed waveguide were carried out to test the feasibility of MMW viscosity measurements. After a few tests with the original $41.3 \mathrm{~mm}$ diameter mullite waveguide it was found that the glass flow rate was too fast to reliably measure viscosities below about 200 Poise due to the time response limits of the data acquisition system being used. The waveguide was changed to a $28.6 \mathrm{~mm}$ diameter mullite tube. With the smaller diameter waveguide viscosities down to about 20 Poise could be resolved. 
The viscosities of the glass compositions used in these tests were obtained from a commercial laboratory ${ }^{2}$. MMW measured flow rates were then compared to these independently obtained viscosities. The flow rates were measured by immersing the waveguide into the glass and then pressurizing the waveguide to displace the molten glass a given distance. The pressure was then quickly released by triggering an exhaust solenoid valve and the time for the glass to flow back up the waveguide was recorded. This was done for more than one displacement and differences in flow time were used for comparison to viscosity to avoid surface tension effects. The furnace temperature was used to vary the glass viscosity.

A glass composition (Hanford \#8) representing Hanford high-level waste (HLW) glass composition was chosen. The target composition (wt.\%) is: $\mathrm{SiO}_{2}=$ $45.14, \mathrm{~B}_{2} \mathrm{O}_{3}=7.03, \mathrm{Na}_{2} \mathrm{O}=20.00, \mathrm{Li}_{2} \mathrm{O}=3.01, \mathrm{CaO}=1.17, \mathrm{MgO}=0.19, \mathrm{Fe}_{2} \mathrm{O}_{3}$ $=14.56, \mathrm{Al}_{2} \mathrm{O}_{3}=6.02, \mathrm{ZrO}_{2}=3.01$, and others $=4.95$. The results are shown in Figure 8. The known viscosity of the glass is plotted as a function of flow time difference between a 0.5 inch and 1.2 inch water pressure displacement. The points are experimental measurements and the curve is a straight line fit to the data on this log-log plot. The measured flow time tracks the viscosity perfectly over a 100:1 dynamic range of 20 to 2000 Poise. Work is currently in progress on viscosity measurements on other glasses and density measurements at high temperature are also being investigated.

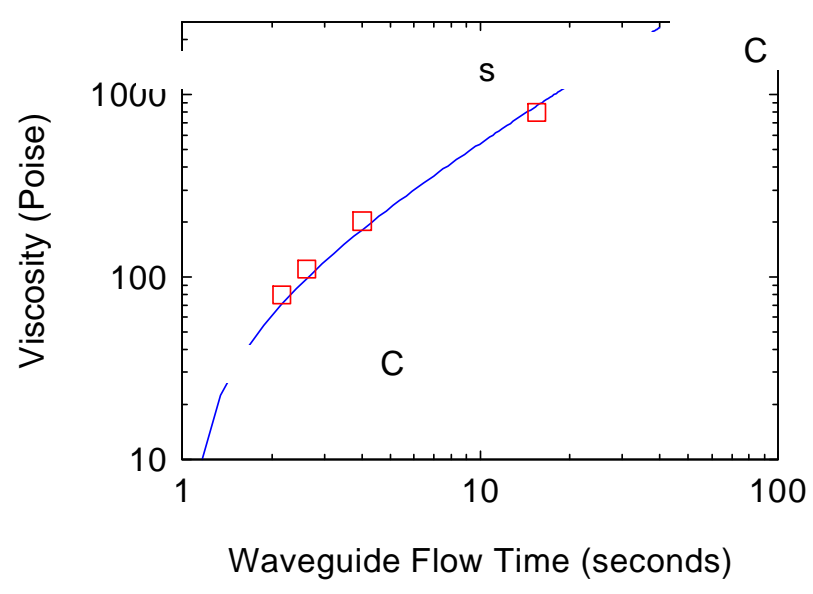

Figure 8. Viscosity versus measured MMW flow time inside a $28.6 \mathrm{~mm}$ diameter waveguide

\footnotetext{
${ }^{2}$ CELS - Corning Laboratory Services, Corning, NY 14831
} 


\section{SUMMARY}

Significant new on-line monitoring capability for glass melters using robust millimeter-wave techniques have been described and tested in the laboratory. The capability to monitor temperature, reflectivity (for emissivity determination), density, and viscosity has been shown and the feasibility in the laboratory demonstrated. Refractory millimeter-wave guides have been developed and used routinely for efficiently interfacing sensitive millimeter-wave electronics with a high temperature furnace in an oxidizing environment. Implementing millimeterwave diagnostics as described here on nuclear waste melters should make possible feedback control of the vitrification process. This would lead to reduced costs and risks in the waste clean up effort.

The monitoring techniques described here are not limited to in applicability only to waste glass melters. They can also be applied to other high temperature materials processes. It is expected there will be a wide interest in these monitoring techniques. Future developments of MMW technologies may also lead to new capabilities for high temperature materials research studies.

\section{ACKNOWLEDGEMENT}

This work was supported by the Environmental Management Science Program, U. S. Department of Energy under grant number DE-FG07-01ER62707.

\section{REFERENCES}

${ }^{1}$ P. P. Woskov, D. R. Cohn, D. Y. Rhee, and P. Thomas, “Active millimeterwave pyrometer", Rev. Sci. Instrum., Vol. 66, 4241-4248 (1995).

${ }^{2}$ S. Ramo, J. R. Whinnery, and T. Van Duzer; p. 291 in Fields and Waves in Communication Electronics, John Wiley \& Sons, New York, 1984.

${ }^{3}$ M. A. Heald and C. B. Wharton; Section 7.2 in Plasma Diagnostics with Microwaves, John Wiley \& Sons, 1965.

${ }^{4}$ J. L. Doane; Vol. 13 in Infrared and Millimeter Waves, Edited by K. Button, Academic Press, 1985. 